Revista Ambientale

Revista da Universidade Estadual de Alagoas/UNEAL

e-ISSN 2318-454X, Ano 13, Vol. 13 (4), 2021

https://doi.org/10.48180/ambientale.v13i4.323

\title{
Mapeamento da vulnerabilidade natural das águas subterrâneas do município de Jaguarão/RS
}

\section{Mapping natural vulnerability of groundwater in the city Jaguarão/RS}

\author{
Nantyara Borges Bandeira ${ }^{1}$, Luana Nunes Centeno ${ }^{2}$, Samanta Tolentino Cecconello ${ }^{3}$
}

1.Instituto Federal de Educação, Ciência e Tecnologia Sul-rio-grandense Câmpus Pelotas, email: nantyaraborgess@gmail.com; 2. Universidade Federal de Pelotas, e-mail:

luananunescenteno@gmail.com; 3. Instituto Federal de educação, Ciência e Tecnologia Sul-riograndense Câmpus Pelotas, e-mail: samantacecconello@ifsul.edu.br

\begin{abstract}
Resumo - As águas subterrâneas apresentam-se como um recurso natural de extrema importância para o abastecimento humano, porém, devido às atividades antrópicas, os mananciais subterrâneos sofrem grandes riscos à contaminação. Deste modo, este estudo teve o objetivo de identificar e mapear a vulnerabilidade natural à contaminação das águas subterrâneas, bem como estimar a tendência da direção de fluxo do município de Jaguarão/RS utilizando o método GOD. Para isso foram utilizados dados de 90 poços cadastrados no CPRM/SIAGAS para o município de Jaguarão sendo aplicada a metodologia GOD para obtenção da vulnerabilidade em cada poço e testou-se dois métodos de interpolação para gerar o mapa de vulnerabilidade natural à contaminação das águas subterrâneas. Utilizou-se a superfície potenciométrica para determinação do sentido e direção de fluxo subterrâneo. Como resultados obtidos, o método da krigagem ordinária foi o que melhor se ajustou aos dados, gerando um mapa com as classes: insignificante, baixa e média vulnerabilidades à contaminação. A tendência de direção de fluxo demonstrou que o fluxo subterrâneo converge para áreas mais subsuperficiais.
\end{abstract}

Palavras-chave: Método GOD. Krigagem ordinária. IDW. Direção de fluxo.

\begin{abstract}
The groundwater is natural resource of extreme importance for human supply. However, due to human activities, groundwater sources suffer great risks of contamination. Thus, this study aimed to identify and map the natural vulnerability to groundwater contamination, as well as estimate the trend of the flow direction in the city of Jaguarão/RS using the GOD method. For this, data from 90 wells registered in the CPRM/SIAGAS for the city Jaguarão were used the GOD methodology to obtain the vulnerability in each well and two interpolation methods were tested to generate the natural vulnerability map to groundwater contamination. The potentiometric surface was used to determine the direction and direction of groundwater flow. As results obtained, the method of ordinary kriging was the one that best fit the data, generating a map with the classes: insignificant, low and medium vulnerabilities to contamination. The flow direction trend demonstrated that the groundwater flow converges to more subsurface areas.
\end{abstract}




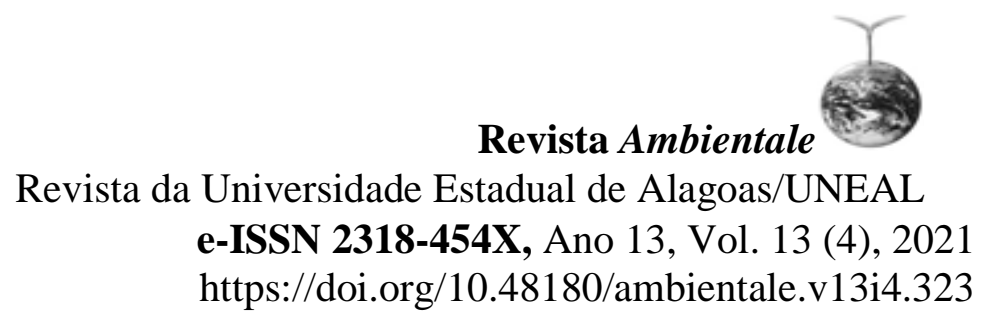

Keywords: GOD method. Ordinary kriging. IDW. Flow direction.

\section{Introdução}

As formações geológicas permeáveis denominadas aquíferos são classificadas em três tipos: fraturado, poroso e cárstico (ANA, 2021). Estes aquíferos podem ser definidos como reservas de água embaixo do solo, abastecidos pelas precipitações (SILVA et al., 2013). Os aquíferos quando submetidos as forças de adesão e de gravidade desempenham um papel fundamental na manutenção da umidade do solo, do fluxo dos rios e lagos (CANOVA; SALAZAR, 2018).

Cabe ressaltar que de acordo com Borges, Athayde e Reginato (2017) a nível mundial, metade da população é abastecida por aquíferos subterrâneos. No Brasil, os aquíferos contribuem para abastecer integralmente $39 \%$ dos municípios, além de auxiliarem na manutenção de boa parte dos rios perenes. O município de Jaguarão, localizado no Rio Grande do Sul, objeto deste estudo, utiliza-se da captação de água subterrânea para abastecimento de toda a população rural (ANA, 2021). Além disto, por se tratar de um município cuja economia baseia-se em atividades agropecuárias, com predomínio de orizicultura, o monitoramento da qualidade das águas subterrâneas se faz necessário (IBGE, 2021).

De acordo com Braga et al. (2015), além do uso e manejo irracional do solo, a exploração excessiva dos recursos hídricos subterrâneos, o descumprimento de normas legais e a urbanização, colocam em risco a qualidade das águas subterrâneas. Sendo assim, faz-se necessário que sejam realizados monitorados periódicos, pois este monitoramento viabiliza estratégias e políticas de proteção e conservação desses recursos (VRBA; SOBLSEK, 1988).

Neste contexto, o mapeamento da vulnerabilidade natural à contaminação de aquíferos pode auxiliar no monitoramento e consequentemente no planejamento e gestão ambiental, servindo como instrumento de tomada à decisão (RIBEIRO, ROCHA, GARCIA, 2011; TANAJURA, LEITE, 2017; FELDKIRCHER, LÖBLER, SILVA, 2014; LÖBLER, BORBA, SILVA, 2021; MACHIWAL et al., 2018). Todavia, a vulnerabilidade natural de aquífero pode ser compreendida como a maior ou menor capacidade de um aquífero em ser propenso à contaminação por uma carga poluidora (BROLLO; VELLO; ODA, 2000).

Para o mapeamento das vulnerabilidades das águas subterrâneas, empregam-se técnicas para que cruzam diferentes características naturais do solo, de modo que, quando este for adversamente afetado por uma grande quantidade de poluentes, possa retratar uma maior ou menor resistência a estas modificações, indicando dessa forma uma determinada vulnerabilidade natural à contaminação (FELDKIRCHER, LÖBLER, SILVA, 2014). Com o intuito de avaliar o grau de vulnerabilidade de aquíferos, alguns métodos foram criados com o objetivo de estabelecer categorias de vulnerabilidade: GOD (FOSTER; HIRATA, 1988), DRASTIC (ALLER et al., 1987), AVI (VAN STEMPVOORT et al., 1992), SI (FRANCÉS et al., 2001), EPPNA (1998) e SINTACS (CIVITA, 1994), COP (VÍAS et al. 2006) e POSH (FOSTER et al. 2002; 2006). 


\section{Revista Ambientale \\ Revista da Universidade Estadual de Alagoas/UNEAL \\ e-ISSN 2318-454X, Ano 13, Vol. 13 (4), 2021 \\ https://doi.org/10.48180/ambientale.v13i4.323}

Com diversas aplicações, a saber: o método de POSH (Pollutant Origem Sucharge Hidraulically) que apresenta de forma simplificada a classificação de potenciais contaminantes, foi empregado, por exemplo, para a avaliar a vulnerabilidade de um aquífero da Ilha de Cotijuba por Cardoso et al. (2018). Por conseguinte, os métodos COP (Flow Concentration, Overlying layers, Precipitation) e PI (Protective cover, Infiltration conditions) que são especificamente usados para ambientes que possuem características cársticas, sendo o método COP usado no estudo de vulnerabilidade do sítio cárstico de Shimbar (BAGHERZADEH et al., 2018). Ademais, tem-se o supracitado método DRASTIC (Depth to water, net Recharge, Aquifer media, Soil media, Topography, impacto vadoze zone media, hydraulic Conductivity of the aquifer) que considera a dinâmica de movimentação dos poluentes, utiliza-se um elevado número de parâmetro, $o$ que em muitos casos inviabiliza sua aplicação (SHAHAB et al., 2018).

O GOD, é um dos sistemas mais empregados por necessitar de um menor conjunto de parâmetros, a saber: tipo de ocorrência do aquífero, litologia das camadas rochosas e profundidade do aquífero (FOSTER; HIRATA, 2002). Com relação as suas vantagens estes modelos apresentam praticidade e qualidade o que o distingue de outros métodos. Desta forma, o método GOD (Groundwater hydraulic confinement, Overlaying strata, Depth to groundwater table) é amplamente adotado no Brasil, pois além do comentado anteriormente, ele utiliza informações que já estão no sistema de regularização de poços do SIAGAS (GHAZAVI; EBRAHIMI, 2015; GARCIA; CONCEIÇÃO; PEIXOTO, 2018; LASAGNA; LUCA; FRANCHINO, 2018). O método GOD assim como outros métodos de vulnerabilidade à contaminação das águas subterrâneas, necessita de um ambiente de Sistema de Informações Geográficas - SIG para espacializar os parâmetros e interpolar os valores para toda a área de estudo (DUHALDE et al., 2018; BORBA et al., 2016; MEIRA, CAMPOS, PEREIRA, 2014).

Juntamente com o mapeamento da vulnerabilidade à contaminação das águas subterrâneas, as estimativas das direções de fluxos, são de grande importância para a gestão e o planejamento. Dentro deste contexto, a direção de fluxo refere-se as associações hidrológicas entre posições diferentes dentro de uma bacia hidrográfica (SOUZA et al., 2013). As redes de fluxo são ferramentas analíticas mais desenvolvidas para a pesquisa do fluxo de água subterrânea em concordância com Freeze e Cherry (2016), para isto de suma importância atrelar o mapeamento da vulnerabilidade natural com a direção de fluxo (PINHEIRO et al., 2015). Diante do exposto, este estudo objetivou identificar e mapear a vulnerabilidade natural à contaminação das águas subterrâneas através do método GOD, bem como estimar a tendência da direção de fluxo do município de Jaguarão/RS.

\section{Material e Métodos}

\section{Caracterização da Área}

O município de Jaguarão está situado no extremo meridional do Brasil (Figura 1), fronteira com a República Oriental do Uruguai, a uma distância de aproximadamente 388 km da capital do estado, ou seja, Porto Alegre. Possui uma população estimada de 27931 habitantes, conforme o último censo realizado em 2010 (IBGE, 2010). Jaguarão tem um 


\section{Revista Ambientale \\ Revista da Universidade Estadual de Alagoas/UNEAL \\ e-ISSN 2318-454X, Ano 13, Vol. 13 (4), 2021 \\ https://doi.org/10.48180/ambientale.v13i4.323}

território cuja extensão total é de 2051,021 km², com as coordenadas de latitude 32³3'58" Sul e a uma longitude 5322'33" Oeste, estando a uma altitude de 26 metros.

Segundo dados da Agência Nacional de Águas e Saneamento (ANA, 2021) o abastecimento da cidade de Jaguarão é realizado pelo rio Jaguarão e a distribuição de água é realizada pela Companhia Riograndense de Saneamento - CORSAN no perímetro urbano. Na zona rural o abastecimento de água se dá por captações de água subterrânea através de poços tubulares e de cacimbas. De acordo com o Sistema de Águas Subterrâneas - SIAGAS (2021), os poços tubulares regularizados são 90 (SIAGAS, 2021).

Figura 1. Localização da área de estudo.

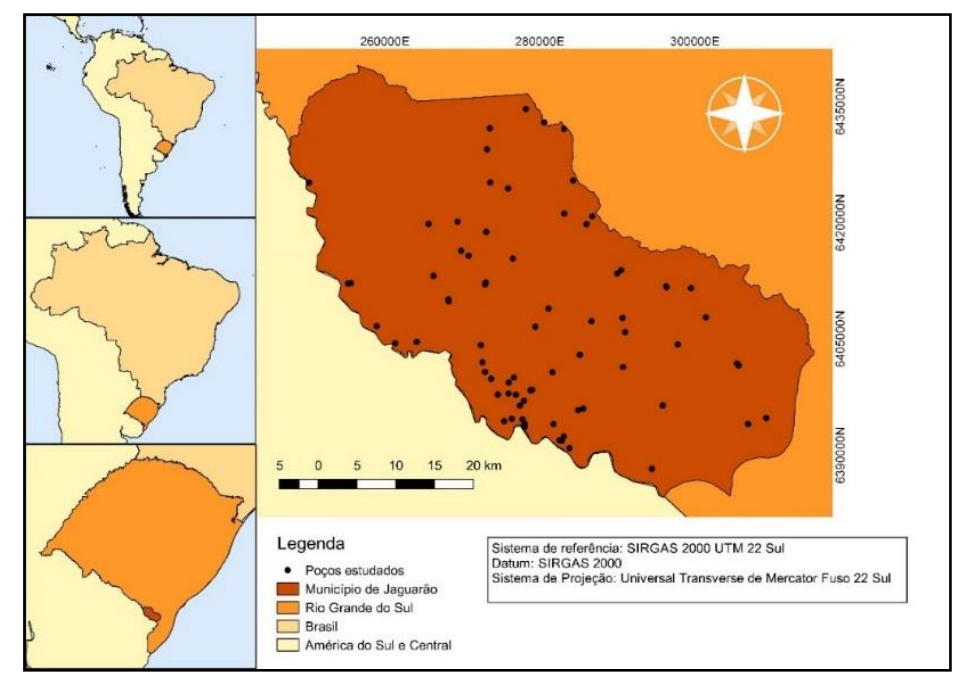

A economia baseia-se principalmente no setor da agricultura e pecuária, mas também no turismo, por conta dos sítios arqueológicos, e o comércio por sua localização próximas aos Freeshops no Uruguai. Destaca-se que a produção de arroz é uma das principais do município atingindo 256.020,549 toneladas de grãos, enquanto a quantidade de soja produzida é de 129.097,678 toneladas (IBGE, 2021).

Segundo dados do Serviço Geológico do Brasil (CPRM, 2006) o município de Jaguarão encontra-se em grande parte territorial, inseridas no Complexo GranitoGnaíssico, outra parte se fragmenta em depósitos litorâneos e formação Serra Geral. Os principais usos dos poços são para abastecimento doméstico, animal e irrigação, onde o nível estático dos poços cadastrados junto ao SIAGAS varia de 1 metros a 180 metros. No município o solo é formado principalmente por solos do tipo Planossolos Háplicos Eutróficos, Gleissolos Háplicos Eutróficos, Neossolos Flúvicos Eutróficos, Neossolos Flúvico Eutróficos e Argilossolos Vermelho-Amarelo Distrófico (IBGE, 2021).

Os planossolos, gleissolos, neossolos, tem baixa permeabilidade com severo acúmulo de argila em superfície. São de baixa permeabilidade na superfície, extremamente duros quando seco, condicionam ciclos de redução e oxidação do ferro, ocasionando as cores acinzentadas ou mosqueados, ocorrem em áreas de relevo plano ou suave (SANTOS et al., 2018). Os Argilossolos Vermelho-Amarelo Distrófico distinguidos pelo maior teor de argila nos horizontes subsuperficiais quanto os superficiais. Os minerais predominantes são argilas de atividade baixa (caulita) e/ou óxidos que contribuem para o valor de capacidade de troca catiônica. Esses solos 


\section{Revista Ambientale \\ Revista da Universidade Estadual de Alagoas/UNEAL \\ e-ISSN 2318-454X, Ano 13, Vol. 13 (4), 2021 \\ https://doi.org/10.48180/ambientale.v13i4.323}

apresentam uma cor acinzentada a avermelhada sendo matizes amarelos e vermelhos os mais comuns (LUMBRERAS et al., 2018).

\section{Compilação dos Dados}

Os dados foram obtidos através de informações contidas no sítio SIAGAS (Sistema de Informações de Águas Subterrâneas) mantido pela CPRM (Companhia de Pesquisa de Recursos Minerais) para o município de Jaguarão. Foram contabilizados 90 poços cadastrados, dos quais foram extraídas informações sobre cada poço, com o objetivo de criar um banco de dados espaciais. Foram obtidas informações sobre as coordenadas geográficas, nível dinâmico, nível estático, cota do terreno, formação geológica, litologia, grau de confinamento e situação atual de operação do poço.

\section{Aplicação do Método GOD}

A metodologia GOD, proposta por Foster et al. (2006) e foi aplicada para a avaliação da vulnerabilidade natural à contaminação das águas subterrâneas do município de Jaguarão/RS, que leva em conta a avaliação de três parâmetros, a saber:

Ocorrência de água subterrânea (Groundwater occurrence, parâmetro G): leva em consideração o tipo de aquífero, onde os valores variam entre 0 a 1 . Observamos o grau de confinamento do aquífero, onde ele poder ser: nenhum, confinado, semi-confinado, não confinado (coberto), e não confinado.

Classificação dos extratos acima da zona saturada de um aquífero (Overall aquifer class, parâmetro O): condicionam o tempo de deslocamento de contaminantes, onde são atribuídos valores entre 0,4 e 1,0. Esta fase trata da determinação dos tipos litológicos no alto da zona saturada do aquífero, com a diferenciação do grau de consolidação e das propriedades de granulometria. Por exemplo, se pela natureza litológica for caracterizada por sedimentos arenosos inconsolidados de origem eólica será dado o valor de 0,6 .

E o terceiro parâmetro $(\mathrm{P})$ profundidade do topo do aquífero (Depth to groundwater), que é a distância que o contaminante terá que percorrer para chegar na zona saturada do aquífero, variando os valores entre 0,6 a 1 . A profundidade da água do aquífero, refere-se a distância que o contaminante terá que percorrer para atingir a zona saturada do aquífero. Para distâncias maiores de 50 metros o valor será de 0,6; para distâncias de 20 a 50 metros, valor de 0,7; para distâncias de 5 a 20 metros o valor de 0,8; para distâncias menores de 5 metros dado o valor de 0,9 . Por exemplo, se o aquífero tem uma distância de 25 metros é dada a pontuação de 0,7 .

Assim após contabilizar todos os pontos, multiplica-se o valor atribuído ao tipo de aquífero $(\mathrm{G})$ pelo valor atribuído a litologia $(\mathrm{O})$, o resultado será multiplicado pelo valor atribuído a distância da água (D). O resultado será a vulnerabilidade, que é dividida em classes que correspondem ao grau de vulnerabilidades, em que são classificadas da seguinte forma: a) Extrema: valores entre 0,7 a 1,0; alta: valores entre 0,5 a 0,7; média: valores entre 0,3 a 0,5 ; baixa valores entre 0,3 a 0,5 ; e insignificante, quando apresentar valores entre 0 a 0,3. A Figura 2 demonstra a metodologia GOD proposta por Foster et al. (2006). 
Revista Ambientale

Revista da Universidade Estadual de Alagoas/UNEAL

e-ISSN 2318-454X, Ano 13, Vol. 13 (4), 2021

https://doi.org/10.48180/ambientale.v13i4.323

Figura 2. Esquema método GOD.

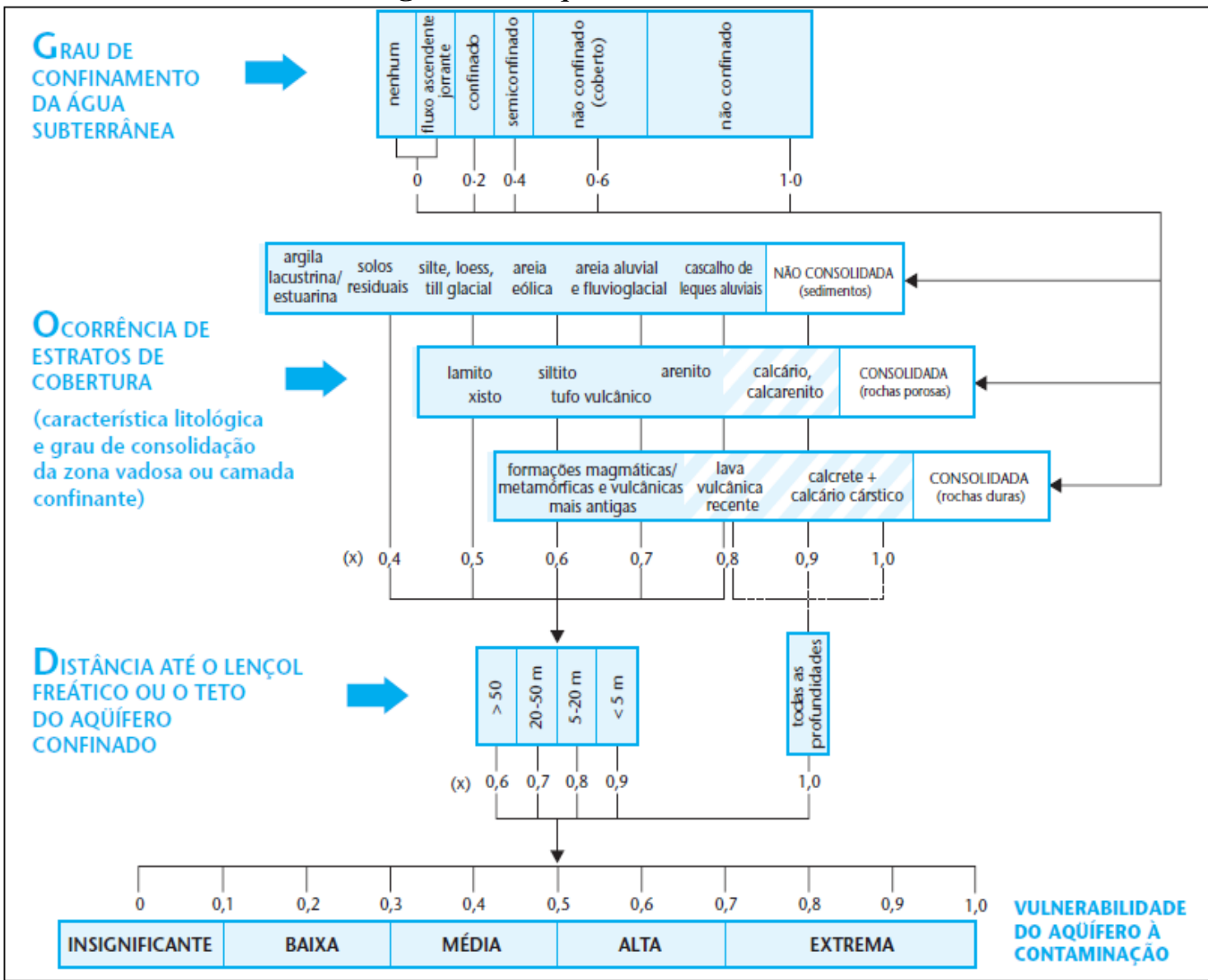

Fonte: Foster et al. (2006)

O método aplica constantes de 0 e 1 para cada variável, sendo assim todos os parâmetros possuem o mesmo nível de importância. Com isso, o índice de vulnerabilidade é obtido com a multiplicação dos valores de cada fator, variando de vulnerabilidade insignificante $(0,0)$ até vulnerabilidade extrema $(1,0)$.

\section{Método de Interpolação}

Para estimar os valores do GOD em pontos não amostrados, foi realizada a interpolação dos dados através da ferramenta denominada Geostatistical Analyst e Inverse Distance Weighting - IDW, para fins de comparação, por meio do software ArcGIS Desktop ${ }^{\circledR}$ versão 10.1, através do método de Krigagem Ordinária e do Inverso da Distância. 


\section{Revista Ambientale}

Revista da Universidade Estadual de Alagoas/UNEAL

e-ISSN 2318-454X, Ano 13, Vol. 13 (4), 2021

https://doi.org/10.48180/ambientale.v13i4.323

\section{Superfície Potenciométrica e Direção de Fluxo}

Para facilitar a identificação da direção das plumas de contaminação utilizou-se o estudo de tendência de direção de fluxo das águas subterrâneas do município. A tendência de direção de fluxo foi obtida através do mapa da superfície potenciométrica. A superfície potenciométrica foi obtida com base na diferença entre a cota da "boca" do poço, extraída do modelo digital de elevação e o nível estático da água de cada poço avaliado, extraída da base de dados do SIAGAS. A espacialização dos dados foi realizada no ambiente QGIS 2.8 sendo o método de interpolação da superfície potenciométrica foi a krigagem ordinária.

\section{Resultados e Discussão}

Através da análise dos 90 poços utilizados neste estudo, foi possível caracterizar os mesmos quanto aos critérios utilizados para obter a vulnerabilidade natural à contaminação das águas subterrâneas, conforme pode-se observar na Figura 3.

Figura 3. Porcentagem dos valores atribuídos a cada um dos parâmetros do método GOD para os poços do município de Jaguarão/RS.

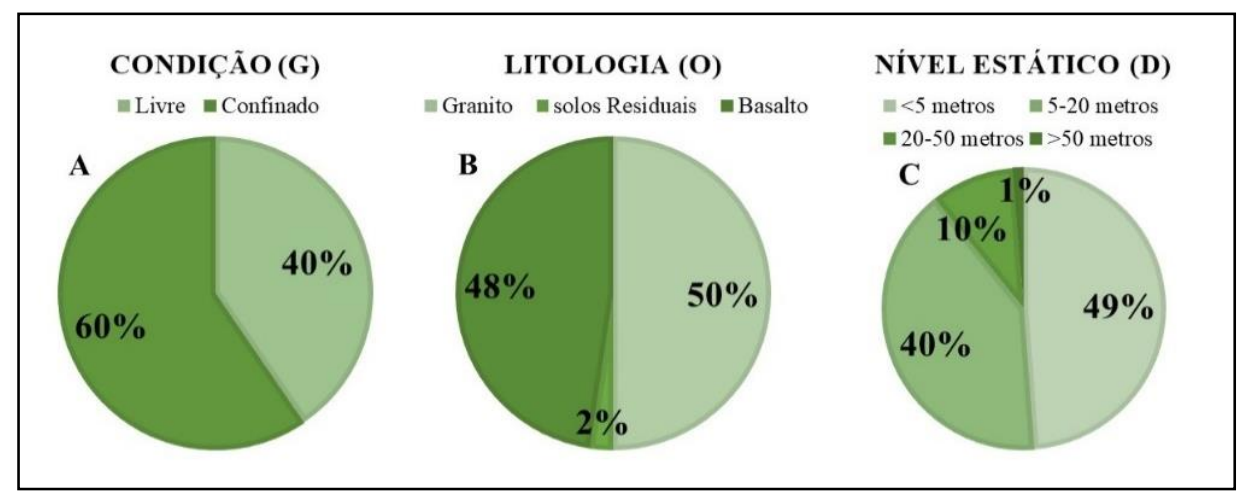

De acordo com a Figura 3A, 60\% dos poços foram classificados como confinados e $40 \%$ como livre. Percebe-se que 54 poços apresentaram vulnerabilidade baixa no parâmetro $\mathrm{G}$ e estes poços receberam peso 0,2 . Já os 36 poços restantes apresentaram alta vulnerabilidade, recebendo peso 1 .

Já com relação à litologia, observa-se que $50 \%$ dos poços analisados, apresentam a ocorrência de extrato de cobertura de formação granítica, $48 \%$ são solos residuais e apenas $2 \%$ são classificados como basalto. A formação granítica, apresentam material proveniente de rochas magmáticas e que por sua vez são mais duras, resistentes e apresentam baixa permeabilidade (PRESS et al., 2006). Já os solos residuais, também denominados de solos saprolíticos, são compostos por grãos, agregados e cristais de minerais alterados, grumos de argilominerais e partículas de resistências variadas, com 


\section{Revista Ambientale \\ Revista da Universidade Estadual de Alagoas/UNEAL \\ e-ISSN 2318-454X, Ano 13, Vol. 13 (4), 2021 \\ https://doi.org/10.48180/ambientale.v13i4.323}

tendência à quebra e/ou desarranjo quando submetidos a pressões, sendo na maioria das vezes bem mais frágeis que os solos graníticos.

Com relação à distância do lençol freático ou o teto do aquífero confinado, $49 \%$ dos poços avaliados encontram-se a uma distância menor que 5 metros, já $40 \%$ dos poços encontram-se entre 5 e 20 metros de profundidade, $10 \%$ e $1 \%$ encontram-se respectivamente entre 20 e 50 metros e acima de 50 metros de profundidade. Observa-se que quase metade dos poços encontram-se muito próximos da subsuperfície do solo, conferindo um alto grau de vulnerabilidade à contaminação. Com a multiplicação dos três parâmetros resultou em quatro classes de vulnerabilidade natural à contaminação das águas subterrâneas para cada poço: insignificantes, Baixos, Médios e Altos. A classe insignificante representa $37 \%$ da área total do município, a classe média representa $32 \%$, seguida da classe baixa (30\%) e apenas $1 \%$ da área total do município apresentou vulnerabilidade alta. Portanto, observa-se que a maior parte dos poços apresentou vulnerabilidade insignificante (37\%) e baixa (30\%), que somadas representam $67 \%$ dos poços do município. Desta forma, pode-se inferir que a vulnerabilidade natural à contaminação das águas subterrâneas do município de Jaguarão não confere aparentemente grandes preocupações.

O trabalho de Sales et al. (2017), quando avaliaram a vulnerabilidade natural de um aquífero livre que recarrega um dos principais mananciais de abastecimento da cidade de Campo Grande - MS, por meio do modelo GOD, encontraram duas classes no sistema aquífero, apresentando áreas de média e alta vulnerabilidade. Já Amaral e Bayer (2015), quando aplicaram o método GOD em uma sub-bacia do Rio Vermelho localizada em um município de Goiás, encontraram as classificações média e insignificante, sendo que, as áreas mais vulneráveis foram aquelas onde os poços são mais rasos e a litologia formada por regiões de rochas sedimentares.

Silva, Rizzo e Cabral (2021), utilizaram a metodologia GOD como ferramenta para auxiliar na alocação de recursos e na priorização das atividades de monitoramento ambiental e verificaram que os resultados apresentaram que, cerca de 94,76\% da área estudada foram classificadas como de vulnerabilidade alta e 5,26\% de vulnerabilidade média. Os autores justificaram a natureza livre do aquífero e sua constituição arenosa, foi o fator predominante para a variação de vulnerabilidade alta e média encontrada na área de estudo.

Após aplicar a metodologia GOD nos poços cadastrados no SIAGAS para Jaguarão e realizada a interpolação da vulnerabilidade do aquífero por meio de krigagem, o semivariograma experimental identificou a existência de dependência espacial nos dados analisados. Dentre os modelos teóricos existentes, o que melhor se ajustou ao semivariograma experimental foi o exponencial com um alcance máximo de $702,55 \mathrm{~m}$, efeito pepita de 0,00 e patamar de 0,0108. A validação cruzada resultou em erro médio quadrático de 0,1097 , erro médio quadrático padronizado de 1,33 , um erro médio padronizado de 0,0048 e um erro médio de 0,102 .

Cabe destacar que de acordo com Jakob e Young (2006), para que o mapa de predição encontrado apresente valores estimados com qualidade, o erro médio padronizado dos valores estimados deve ser próximo de 0 , que o erro quadrático médio seja o mais baixo possível, que o erro médio seja próximo do erro quadrático médio, e que o erro quadrático médio padronizado seja próximo de 1. Desta maneira, pode-se assumir que o mapa de predição da vulnerabilidade encontra-se adequado. Os resultados 


\section{Revista Ambientale}

Revista da Universidade Estadual de Alagoas/UNEAL

e-ISSN 2318-454X, Ano 13, Vol. 13 (4), 2021

https://doi.org/10.48180/ambientale.v13i4.323

obtidos no mapa de vulnerabilidade para o município de Jaguarão variaram de vulnerabilidade insignificante a média, sendo que em grande parte do município o lençol freático apresentou-se como de baixa vulnerabilidade natural à contaminação, como pode ser observado na Figura 4.

Percebe-se que através do interpolador da krigagem, a vulnerabilidade considerada como alta localmente foi substituída pela média, tendo em vista que a krigagem utiliza a variância espacial dos dados.

Através do mapa de krigagem conforme a Figura 4 a região sul - sudeste do município de Jaguarão apresenta áreas com vulnerabilidade média (corresponde a 10,4\% da área do município), sendo que nesta região tem-se a maior área de uso do solo com pecuária seguido da produção de arroz irrigado e que esta atividade gera grandes problemas como a saturação do solo, e a condução de agrotóxicos, fungicidas e herbicidas para a parte mais profunda do solo (BARRIGOSSI; LANNA; FERREIRA, 2004).

Figura 4. Mapa da krigagem com os dados dos poços especializados para o município de Jaguarão/RS.

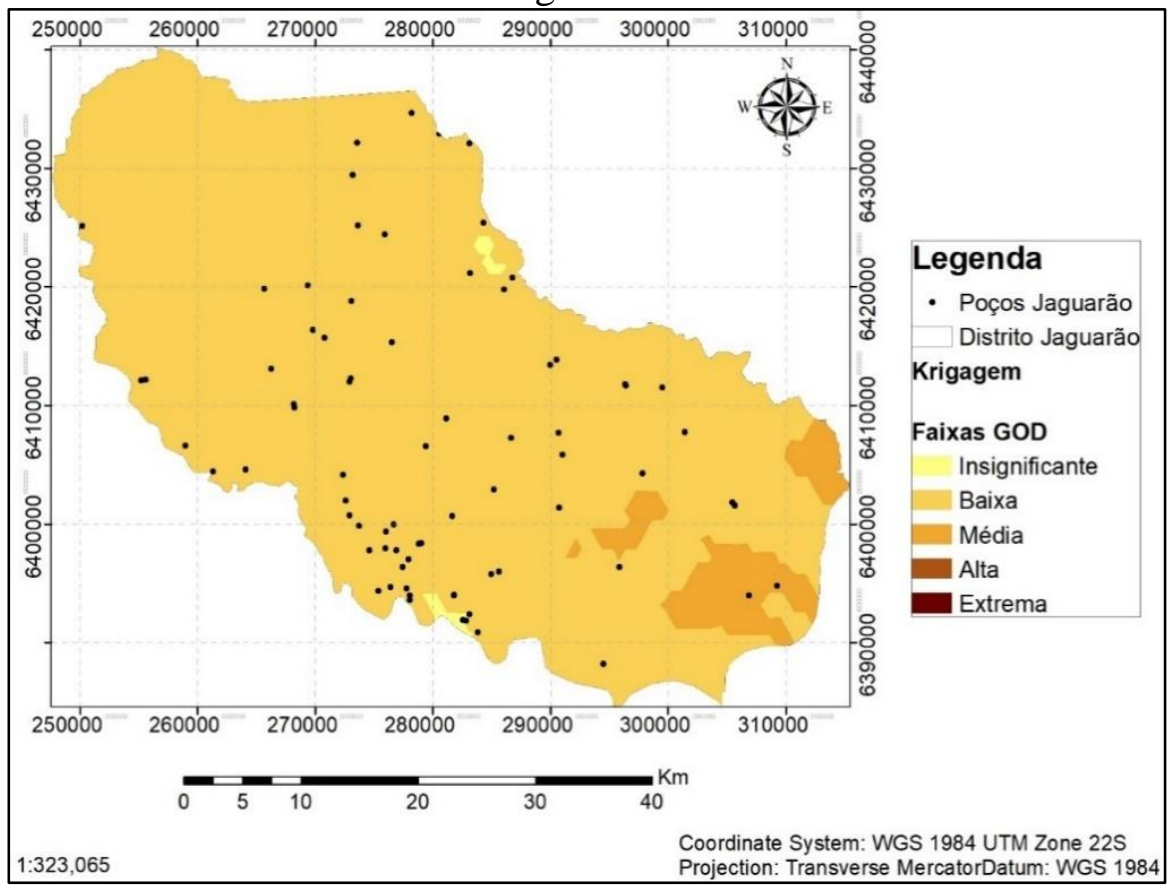

Há duas áreas do município de Jaguarão que apresentaram vulnerabilidade insignificante, localizado no norte e sudoeste do município que juntas somam 1,7\% da área do município. Ademais, o restante do município apresentou uma baixa vulnerabilidade, correspondendo a $87,9 \%$ da área do município, e possui um uso de solo voltado para ocupações de pecuária, cultivos de soja, trigo e milho. Meira et al., (2014) afirmam que a cultura da soja tem grande presença de herbicidas, inseticidas e fungicidas que com a soma de agrotóxicos e metais pesados presentes nos fertilizantes podem contribuir para a poluição do solo.

Já por meio da interpolação do IDW, Figura 5, a região central apresentou vulnerabilidade alta. Porém, de acordo com Yamamoto (2020), este método possui uma 
Revista Ambientale

Revista da Universidade Estadual de Alagoas/UNEAL

e-ISSN 2318-454X, Ano 13, Vol. 13 (4), 2021

https://doi.org/10.48180/ambientale.v13i4.323

propriedade indesejável, que é a formação de regiões planas em torno dos poços, além de considerar apenas a distância e excluir a direção na estimativa, estes fatores podem estar relacionados a área de alta vulnerabilidade encontrada na parte central do mapa.

Figura 5. Mapa IDW com os dados dos poços especializados para o município de Jaguarão/RS.

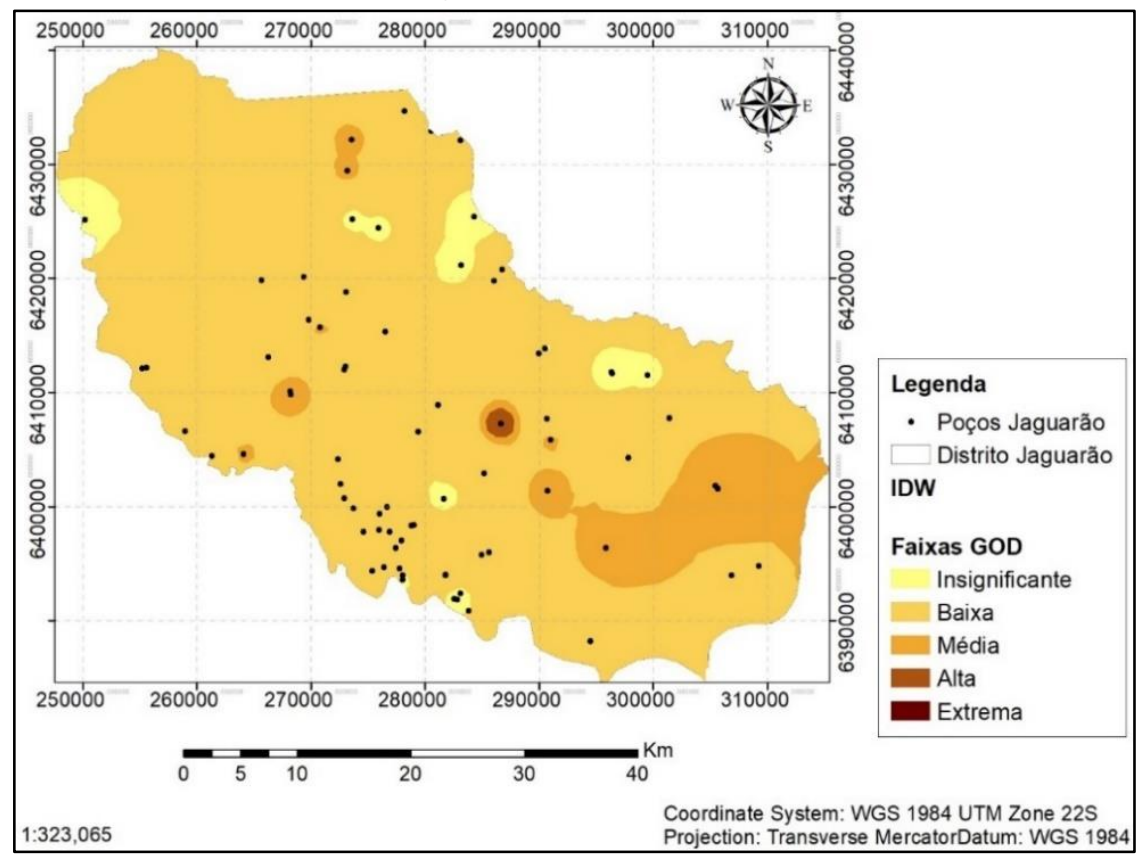

Comparativamente o melhor método de interpolação para determinação da vulnerabilidade natural à contaminação das águas subterrâneas de Jaguarão foi a Krigagem, pois ela é mais robusta que o IDW e apresentou métricas estatísticas satisfatórias para a eficiência do interpolador. Além disso, a krigagem possibilita a análise da variabilidade espacial considerando além da distância de dependência, a sua direção. Deste modo, este método conserva a relação existente entre os poços mais próximos, mostrando assim um mapa mais fidedigno da realidade quando comparado ao interpolador IDW, uma vez que este realiza uma interpolação global, ou seja, ignora as relações de dependência existe entre os parâmetros analisados (YAMAMOTO, 2020). A Figura 6 ilustra o mapa de tendência de direção de fluxo, onde é possível observar a predominância de fluxo de áreas de elevada superfície potenciométrica $(143,65 \mathrm{~m})$ para áreas de baixa superfície potenciométrica $(5,51 \mathrm{~m})$. 
Revista Ambientale

Revista da Universidade Estadual de Alagoas/UNEAL

e-ISSN 2318-454X, Ano 13, Vol. 13 (4), 2021

https://doi.org/10.48180/ambientale.v13i4.323

Figura 6. Mapa de tendencia de direção de fluxo para o município de Jaguarão/RS.

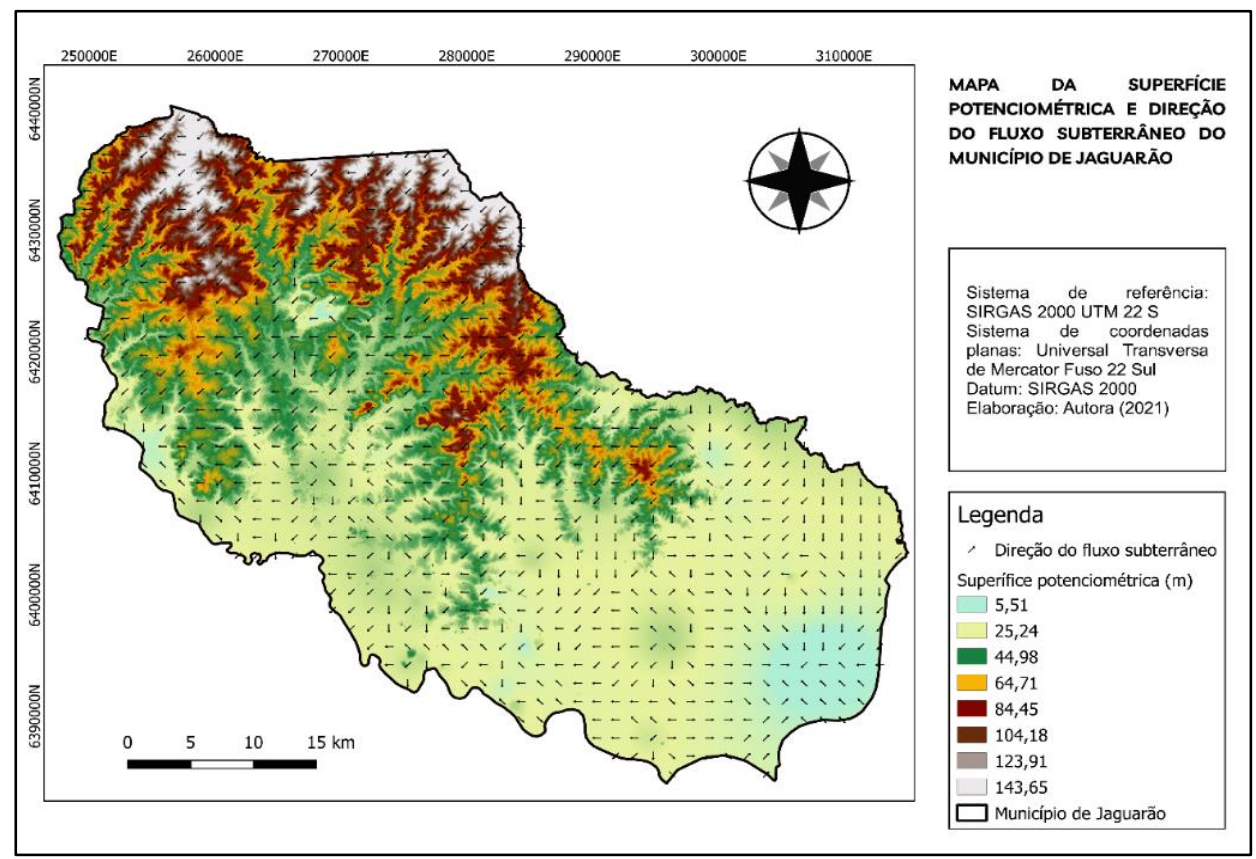

A predominância desse comportamento de fluxo é preocupante, pois predominantemente o fluxo subterrâneo converge para áreas mais subsuperficiais que por conseguinte apresentam maior propensão a contaminação das águas subterrâneas. Neste caso, como é uma área com menor profundidade, caso ocorra uma contaminação do solo por alguma atividade, o fluxo tende a diluir a contaminação e/ou direcionar a mesma para os recursos hídricos. Deste modo, é de suma importância o planejamento e gestão do uso e ocupação da área pelos órgãos gestores, bem como análises mais específicas para avaliar e proteger as áreas de captação de água no município de Jaguarão.

\section{Conclusão}

Conclui-se com este estudo que através da aplicação do método GOD em 90 poços cadastrados pelo SIAGAS para o município de Jaguarão, foi possível testar dois interpoladores (krigagem ordinária e IDW) para mapear a vulnerabilidade à contaminação das águas subterrâneas no município.

Como resultado desta aplicação, verificou-se que o método da krigagem foi o mais adequado para a área de estudo e que através dela obteve-se três classes de vulnerabilidade para o município: insignificante $(1,7 \%)$, baixa $(87,9 \%)$ e média $(10,4 \%)$.

Pode-se verificar ainda que o estudo da tendência de direção de fluxo atrelada ao estudo de vulnerabilidade natural à contaminação pode auxiliar no planejamento e gestão dos recursos hídricos por parte de órgão de gestão ambiental. 
Revista Ambientale

Revista da Universidade Estadual de Alagoas/UNEAL

e-ISSN 2318-454X, Ano 13, Vol. 13 (4), 2021

https://doi.org/10.48180/ambientale.v13i4.323

\section{Conflite de interesse}

Os autores deste manuscrito não declararam conflitos de interesse.

\section{Referências}

AMARAL, Ana Karolyna Nunes et al. Vulnerabilidade dos aquíferos da sub-bacia do Rio Vermelho: aplicação do método GOD. In: SIMPÓSIO BRASILEIRO DE SENSORIAMENTO REMOTO, 17., 2015, João Pessoa. Anais XVII. São Pessoa: Inpe, 2015. p. 1-8.

ANA - Agência Nacional das Águas ANA (Brasil). Rede nacional: redes de monitoramentos. Brasília: ANA, 2021.

ALLER, L.; BENNET, T.; LEHR, J. H. \& PETTY, R. J. 1987. DRASTIC: A standardized system for evaluating groundwater pollution potential using hydrogeologic settings. United States Environmental Protection Agency. EPA/600/2-85/018. pg. 29.

BAGHERZADEH, Somayeh et al. Groundwater vulnerability assessment in karstic aquifers using COP method. Environmental Science and Pollution Research, [s.1.], v. 25, n. 19, p.18960-18979, 2 maio 2018.

BARRIGOSSI, José Alexandre Freitas; LANNA, Anna Cristina; FERREIRA, Evane.

Agrotóxicos no Cultivo do Arroz no Brasil: análise do consumo e medidas para reduzir o impacto ambiental negativo. Emabrapa - Arroz e Feijão: Circular técnica 67, Santo Antônio de Goiás, p.1-7, dez. 2004.

BORBA, W. F. et al. Assessment of the Intrinsic Vulnerability to Contamination of the Aquifer in River Basin Passo Fundo of Rio Grande do Sul. Anuário do Instituto de Geociências URFJ, [s.l.], v. 39, n. 2, p.145-154, 26 maio 2016.

BORGES, Vinícius Menezes; ATHAYDE, Gustavo Barbosa; REGINATO, Pedro Antônio Roehe. Avaliação da vulnerabilidade natural à contaminação do sistema aquífero Serra Geral no Estado do Paraná Brasil. Águas subterrâneas, [SI], v.31, n.4, p.327-337, 9 de out. 2017.

BRAGA, Benedito et al (org.). Águas doces no Brasil: capita ecológico, uso e conservação. 4. ed. São Paulo: Escrituras, 2015. 730 p.

BROLLO, Maria José; VELLO, Ricardo Vedo; ODA, Geraldo Hideo. Avaliação da vulnerabilidade natural de aquíferos à contaminação através de um sistema gerenciador de informações geoambientais: Um instrumento de gestão ambiental. In: XXVII de Engenharia Sanitária E Ambiental, 2000, Porto Alegre. Anais do XXVII Congresso Interamericano de Engenharia Sanitária e Ambiental. Rio de Janeiro: Abas, 2000. p. 1 - 15.

CANOVA, Angelo Vianei; SALAZAR, Rodrigo Fernando dos Santos. Avaliação da vulnerabilidade á contaminação das águas subterrâneas do município de Ibirubá-RS. Ciência \& Tecnologia - Universidade de Cruz Alta, Cruz Alta, v. 2, n. 1, p.75-88, ago. 2018.

CARDOSO, Raisa Nicole Campos et al. Análise da vulnerabilidade e do risco a contaminação de aquíferos semiconfinados na Ilha de Cotijuba/PA. Águas Subterrâneas, [s.1.], v. 32, n. 2, p.1-9, 25 jun. 2018.

CIVITA, M. Le Carte della vulnerabilità degli acquiferi all inquinamento: Teoria e pratica. Pitágora Editrice, Bologna. 1994.

CPRM - Companhia de Pesquisa de Recursos Minerais. SIAGAS. Sistema de informações de águas subterrâneas. Disponível em: https://cutt.ly/jQnFmDb. Acesso em: 20 jul. 2021. 


\section{Revista Ambientale \\ Revista da Universidade Estadual de Alagoas/UNEAL \\ e-ISSN 2318-454X, Ano 13, Vol. 13 (4), 2021 \\ https://doi.org/10.48180/ambientale.v13i4.323}

DUHALDE, Denisse J. et al. Fuzzy-based assessment of groundwater intrinsic vulnerability of a volcanic aquifer in the Chilean Andean Valley. Environmental Monitoring and Assessment, [s.1.], v. 190, n. 7, p.390-404, 11 jun. 2018.

EPPNA, M. Informação cartográfica dos planos de bacia. Sistematização de figuras e cartas a imprimir em papel. Equipa de projeto do plano nacional da água, 1998.29 p.

FELDKIRCHER, Tiago; LÖBLER, Carlos Alberto; SILVA, José Luiz Silvério da. Aplicação do sistema GOD para determinar a vulnerabilidade natural das águas subterrâneas do município de Teutônia (RS). Revista Eletrônica em Gestão, Educação e Tecnologia Ambiental, [s.l.], v. 18, n. 3, p.1137-1145, 1 set. 2014.

FOSTER, S.; HIRATA, R. Groundwater pollution risk assessment: a methodology using available data. WHO-PAHO/HPE-CEPIS Technical Manual, Lima, Peru. 1988. 81p.

FOSTER, S.; HIRATA, R.; GOMES, D.; D'ELIA, M.; PARIS, M. Groundwater quality protections: a guide for Waters ervice companies, municipal authorities and environment agencies. Word Bank, GWMATE. Washington, 2002, 101p.

FOSTER, S.; HIRATA, R.; GOMES, D.; D'ELIA, M.; PARIS, M. Proteção da qualidade da água subterrânea. Um guia para empresas de abastecimento de água, órgãos municipais e agências ambientais. Banco Mundial, Servmar e Engenharia. Versão em Português, 2006. P. 106.

FRANCÉS, A., PARALTA, E., FERNANDES J. RIBEIRO, L. Development and application in the Alentejo region of a method to assess the vulnerability of groundwater to diffuse agriculture pollution: The Susceptibility Index. Lisboa, Geosystem Center IST, Third International Conference on Future Groundwater Resources at Risk. 2001.

FREEZE, Allan R.; CHERRY, John A. Redes de Fluxo. Groundwater. Canadá: Editora Instituto água Sustentável, 2016. Cap. 5. p. 190-215.

GARCIA, Claudinei; CONCEIÇÃO, Fabiano Tomazini da; PEIXOTO, Anna Silvia Palcheco. Vulnerabilidade intrínseca dos aquíferos no município de Piracicaba, através do método GOD. Águas Subterrâneas, [s.1.], v. 32, n. 2, p.218-227, 11 jun. 2018.

GHAZAVI, R.; EBRAHIMI, Z. Assessing groundwater vulnerability to contamination in an arid environment using DRASTIC and GOD models. International Journal of Environmental Science and Technology, [s.1.], v. 12, n. 9, p.2909-2918, 1 maio 2015.

GUERRERO, João Vitor Roque et al. Carta de Fragilidade Ambiental como instrumento de planejamento e conservação de unidades aquíferas: o caso da bacia do rio Clarinho, SP.

Caderno de Geografia, Londrina, v. 28, n. 53, p.385-403, mar. 2018.

IBGE - Instituto Brasileiro de Geografia e Estáticas - Cidades: Jaguarão.

https://cidades.ibge.gov.br/brasil/rs/jaguarao/pesquisa/24/76693 - Consulta realizada em 29 de outubro de 2021.

IBGE - Instituto Brasileiro de Geografia e Estáticas - Censo demográfico cidades: Jaguarão https://cidades.ibge.gov.br/brasil/rs/jaguarao/pesquisa/24/76693 - Consulta realizada em 29 de outubro de 2010.

JAKOB, Alberto Augusto Eichman; YOUNG, Andrea Ferraz. O uso de métodos de interpolação espacial de dados nas análises sociodemográficas. In: Encontro Nacional de Estudos Populacionais, 15., 2006, Caxambu. ABEP 2006 388. Caxambu: Unicamp, 2006. p. 1 22.

LASAGNA, Manuela; LUCA, Domenico Antonio de; FRANCHINO, Elisa. Intrinsic groundwater vulnerability assessment: issues, comparison of different methodologies and correlation with nitrate concentrations in NW Italy. Environmental Earth Sciences, [s.l.], v. 77, n. 7, p.277-293, abr. 2018.

LINHARES, Franklin Mendonça et al. Avaliação da vulnerabilidade e do risco à contaminação das águas subterrâneas da bacia hidrográfica do rio Gramame (PB). Sociedade \& Natureza,

[s.1.], v. 26, n. 1, p.139-157, jan. 2014. 
Revista Ambientale

Revista da Universidade Estadual de Alagoas/UNEAL

e-ISSN 2318-454X, Ano 13, Vol. 13 (4), 2021

https://doi.org/10.48180/ambientale.v13i4.323

LÖBLER, Carlos Alberto; BORBA, Willian Fernando de; SILVA, José Luiz Silvério da. Vulnerabilidade das águas subterrâneas da bacia do rio Santa Maria, Rio Grande do Sul.

Revista do Centro de Ciências Naturais e Exatas: Ciência e Natura, Santa Maria, v. 38, n. 1, p.84-94, jun. 2015.

LUMBRERAS, José Francisco et al. Definição de solo. In: LUMBRERAS, José Francisco et al. Sistema Brasileiro de Classificação de Solos. 5. ed. Brasília: Embrapa, 2018.

MACHIWAL, Deepesh et al. Assessment and mapping of groundwater vulnerability to pollution: Current status and challenges. Earth-science Reviews, [s.1.], v. 185, p.901-927, out. 2018.

MEIRA, José Carlos Rodrigues; CAMPOS, Alfredo Borges; PEREIRA, Lindolfo Caetano. Avaliação da vulnerabilidade, risco e perigo à contaminação de zona de recarga do aquífero Guarani com aplicação dos métodos GOD e POSH. Águas Subterrâneas, [s.1.], v. 28, n. 1, p.31-46, 15 abr. 2014.

PINHEIRO, Rinaldo et al. Aplicação dos Métodos GOD e POSH para determinação da vulnerabilidade e perigo à contaminação dos aquíferos na cidade de Santa MariaRS/Application of the GOD and POSH methods to determine the vulnerability and contamination risk of aquifers in Santa M. Revista de Gestão de Água da América Latina, [s.l.], v. 12, n. 2, p.61-79, 2015.

PRESS, F., Siever, R., Grotzinger, M., Jordan, T. H. Para entender a terra. (4a ed.), (Rualdo M., Trad.). Porto Alegre: Bookman. 2006.

RAHMATI, Omid; POURGHASEMI, Hamid Reza; MELESSE, Assefa M.. Application of GIS-based data driven random forest and maximum entropy models for groundwater potential mapping: A case study at Mehran Region, Iran. Catena, [s.1.], v. 137, p.360-372, fev. 2016. RIBEIRO, Daniela Menezes; ROCHA, Washington Franca; GARCIA, Antônio Jorge Vasconcellos. Vulnerabilidade natural dos aquíferos à contaminação da sub-bacia do rio siriri, Sergipe. Águas subterrâneas, [s.1.], v. 25, n. 1, p.91-102, 5 abr. 2011.

SALES POLON BATISTA, C.; CHIQUITO GESUALDO, G.; COLLA LEITE, P. C.; LASTORIA, G.; GARCIA GABAS, S.; CAVAZZANA, G. H.; DE MENDONÇA CASADEI, J.; DE SOUZA AZOIA, T. Aplicação do método GOD para avaliação de vulnerabilidade de aquífero livre em bacia hidrográfica. Águas Subterrâneas, [S. l.], 2017. Disponível em: https://aguassubterraneas.abas.org/asubterraneas/article/view/28686. Acesso em: 10 dez. 2020. SANTOS, Humberto Goncalves dos et al. Sistema Brasileiro de Classificação de Solos. 5. ed. Brasília: Empresa Brasileira de Pesquisa Agropecuária - Embrapa, 2018. Classificação do Solo Nomenclatura Pedologia.

SHAHAB, Asfandyar et al. Groundwater vulnerability assessment using GIS-based DRASTIC method in the irrigated and coastal region of Sindh province, Pakistan. Hydrology Research, [s.l.], p.1-20, 18 maio 2018.

SILVA, Lucas de Carvalho; RIZZO, Rafael Alexandre; CABRAL, Glaubert Lucas de Carvalho. Vulnerabilidade à poluição da água subterrânea no núcleo urbano do distrito de monte alto, Arraial do Cabo, RJ. Revista de Ciências Ambientais: RCA (ISSN 1981-8858), Canoas, v. 15, n. 2, p. 01-13, 2021.

SILVA, Wellington Perreira da et al. Monitoramento da salinidade de águas subterrâneas em várzea cultivada com cana-de-açúcar fertirrigada com vinhaça. Revista Brasileira de Engenharia Agrícola e Ambiental, Campina Grande, v. 4, n. 18, p.394-401, nov. 2013. SOUZA, Carlos Henrique Wachholz de et al. Delimitação automática da microbacia hidrográfica do Rio das Lontras, através de dados SRTM. In: Simpósio Brasileiro de Sensoriamento Remoto - SBSR, 2013, Foz do Iguaçu. Anais. Researchgate, 2013. p. 5515 5522.

TANAJURA, Douglas da Silva; LEITE, Claudio Benedito Baptista. Quantificação e avaliação da vulnerabilidade natural a contaminação das águas subterrâneas da sub-bacia hidrográfica Jacaré-Pepira/SP. Águas subterrâneas, São Paulo, v. 1, n. 34, p.1-19, 2017. 
Revista Ambientale

Revista da Universidade Estadual de Alagoas/UNEAL

e-ISSN 2318-454X, Ano 13, Vol. 13 (4), 2021

https://doi.org/10.48180/ambientale.v13i4.323

VAN STEMPVOORT, D, EWERT, L. WASSENAAR, L. 1992. AVI: A method for groundwater protection mapping in the Prairie Provinces of Canada. PPWD Pilot Project. Groundwater and Contaminants Project, Environmental Sciences Division, National Hydrology Research Institute.

VARGAS, Tiago de et al. Aplicação do Interpolador IDW para Elaboração de Mapas Hidrogeológicos Paramétricos na Região da Serra Gaúcha. Scientia Cum Industria, [s.1.], v. 6, n. 3, p.38-43, 25 dez. 2018. Universidade Caixias do Sul.

VÍAS J.M. Proposed method for groundwater vulnerability mapping in carbonate (karstic) aquifers: the COP method-application in two pilot sites in southern Spain. Hydrogeol J 14:6:912-925, 2006.

VRBA, J.; SOBLSEK, P. Groundwater monitoring. En Geology and Environment International Manual in three volumes. UNESCO. UNEP.1988.

YAMAMOTO, Jorge Kazuo. Estatística, análise e interpolação de dados geoespaciais. São Paulo: Gráfica Paulos, 2020. 308 p. 